\title{
Theoretical Study of Quasi-Longitudinal Lamb Modes in SiN/c-AlN Thin Composite Plates for Liquid Sensing Applications ${ }^{\dagger}$
}

\author{
Cinzia Caliendo *, Ennio Giovine and Muhammad Hamidullah \\ Institute of Photonics and Nanotechnologies, National Research Council of Italy, IFN-CNR, Via Cineto \\ Romano 42, 00156 Rome, Italy; ennio.giovine@cnr.it (E.G.); m.hamidullah@ifn.cnr.it (M.H.) \\ * Correspondence: cinzia.caliendo@cnr.it; Tel.: +39-06-4152-2211 \\ + Presented at the 4th International Electronic Conference on Sensors and Applications, 15-30 November \\ 2017; Available online: http://sciforum.net/conference/ecsa-4.
}

Published: 14 November 2017

\begin{abstract}
The propagation of quasi-longitudinal Lamb mode along SiN/c-AlN thin composite plates was modeled and analyzed aimed at the design of a sensor able to detect the parameters change of a liquid environment, such as added mass and viscosity changes. Three modes were identified that have high phase velocity and quite good electroacoustic coupling efficiency: the fundamental quasi symmetric mode ( $\mathrm{qS}_{0}$ ) and two higher order quasi-longitudinal modes ( $\mathrm{qL}_{1}$ and $\mathrm{qL}_{2}$ ) with a dominantly longitudinal displacement component at one plate side. The velocity and attenuation of these modes were calculated for different liquid viscosities, and the gravimetric and viscosity sensitivities of both the phase velocity and attenuation were theoretically calculated.
\end{abstract}

Keywords: higher order Lamb modes; membrane; sensors; liquid environment; longitudinal polarization

\section{Introduction}

The propagation of in-plane polarized Lamb modes along thin piezoelectric plates has been previously reported [1], aiming at the design of electroacoustic devices able to work in liquids. Higher order quasi-longitudinally polarized Lamb modes exist that travel at velocities close to that of the longitudinal bulk acoustic wave propagating in the same direction. They have close-to-zero shear vertical particle displacement component at the surface of the plate contacting the liquid environment, that can prevent acoustic energy leakage into the liquid. Moreover, their high velocity makes them suitable for high operating frequencies. Matlab, Disperse, and COMSOL Multiphysics simulations were used to study the dispersion curves and the acoustic field profile of the fundamental and higher order modes traveling in SiN/AlN thin suspended membranes, for fixed SiN and AlN thicknesses $(200 \mathrm{~nm}$ and $1.4 \mu \mathrm{m})$ and variable wavelength $\lambda$. Two higher order modes, $\mathrm{qS}_{1}$ and $\mathrm{qS} \mathrm{S}_{2}$, were found that are slowly dispersive and have dominant longitudinal particle displacement component, at AlN thickness-to-wavelength ratio halN $/ \lambda=0.8$ and 1.6 respectively. The acoustic field profile of the modes shows that the out-of-plane particle displacement are close to zero at one plate side. The electroacoustic coupling coefficient $\mathrm{K}^{2}$ dispersion curves were studied for four different electroacoustic coupling configurations, based on interdigital transducers (IDTs) positioned at one of the AIN surface, with or without a metal floating electrode at the opposite layer surface. The theoretical gravimetric sensitivity in air and the sensitivity to the density-viscosity product of a Newtonian liquid contacting the device was calculated for the three modes. 


\section{Phase Velocity Dispersion Curves}

Lamb waves are acoustic modes that travel along finite thickness plates. The modes are divided into symmetric and anti-symmetric modes, $S_{n}$ and $A_{n}$, ( $n$ is the mode order) with respect to the midplane of the plate. A single material thin plate, even anisotropic, shows a family of modes dispersion curves with evident flat regions belonging to the symmetric mode type: as an example Figure 1 shows the velocity vs. frequency dispersion curves for a c-AlN plate with thickness $\mathrm{H}$ equal to $1.4 \mu \mathrm{m}$.

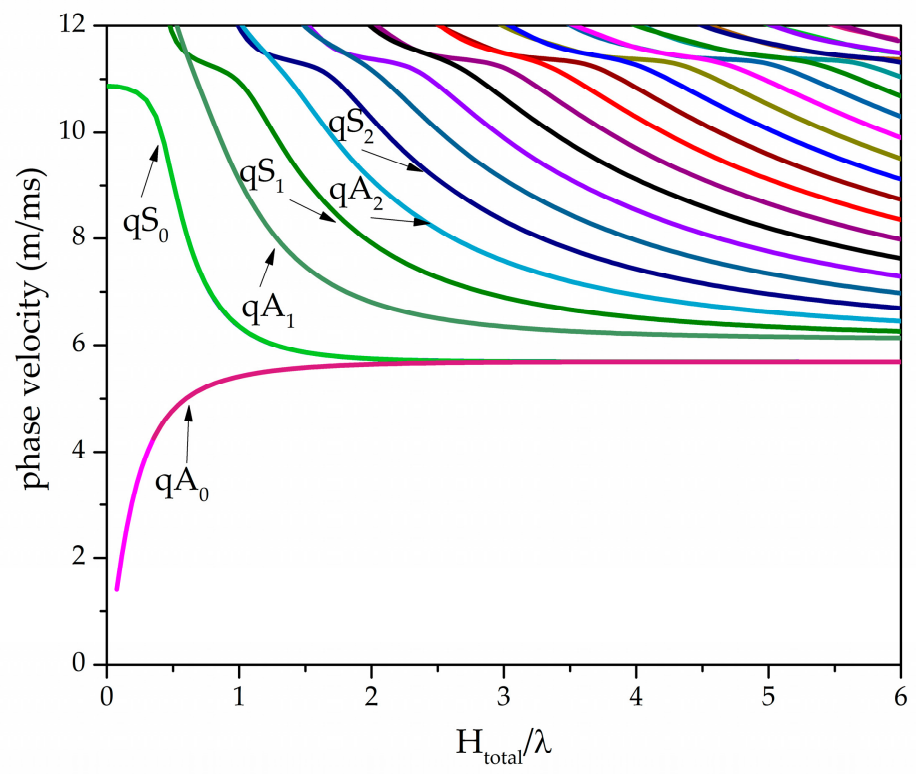

Figure 1. The phase velocity vs. $\mathrm{H} / \lambda$ of the Lamb modes travelling along a c-AlN plate $1.4 \mu \mathrm{m}$ thick in the $\mathrm{x}_{1}$ direction.

Some symmetric modes can be identified that are suitable for liquid sensing applications as the shear vertical $\mathrm{U}_{3}$ displacement component is close to zero at both sides of the symmetric plate. Figure 2a-d show, as an example, the field profile of the first four quasi symmetric modes, $\mathrm{qS}_{0}, \mathrm{qS}_{1}, \mathrm{qS}_{2}$ and qS 3 : these modes are symmetric as the longitudinal component $\mathrm{U}_{1}$ is symmetric with respect to the mid-plane of the plate, while $U_{3}$ is antisymmetric. The two particle displacement components $U_{1}$ and $\mathrm{U}_{3}$ are normalized at the $\mathrm{U}_{1}$ value at the free AlN side; the shear horizontal displacement component $\mathrm{U}_{2}$ is zero.

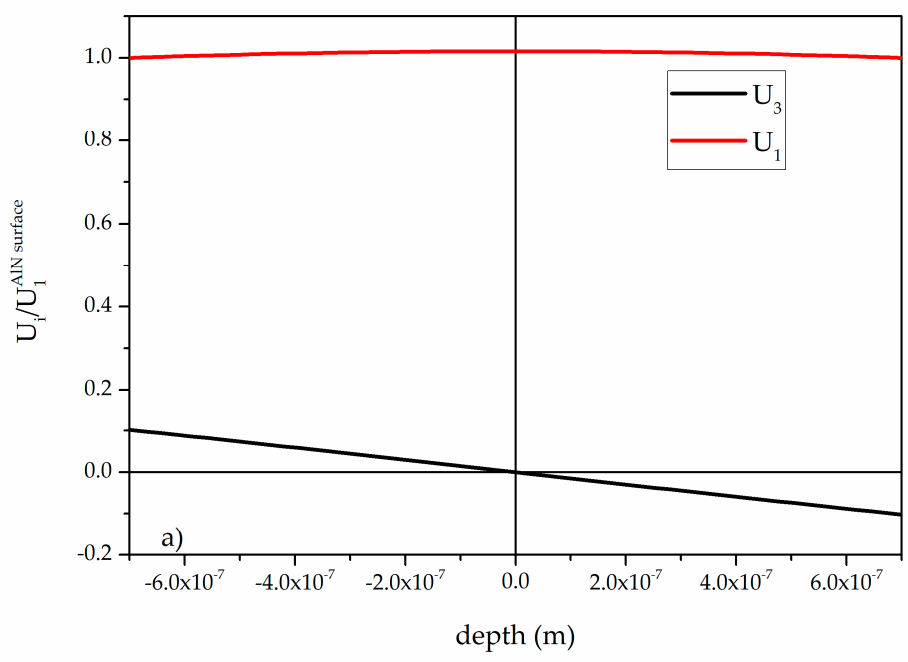



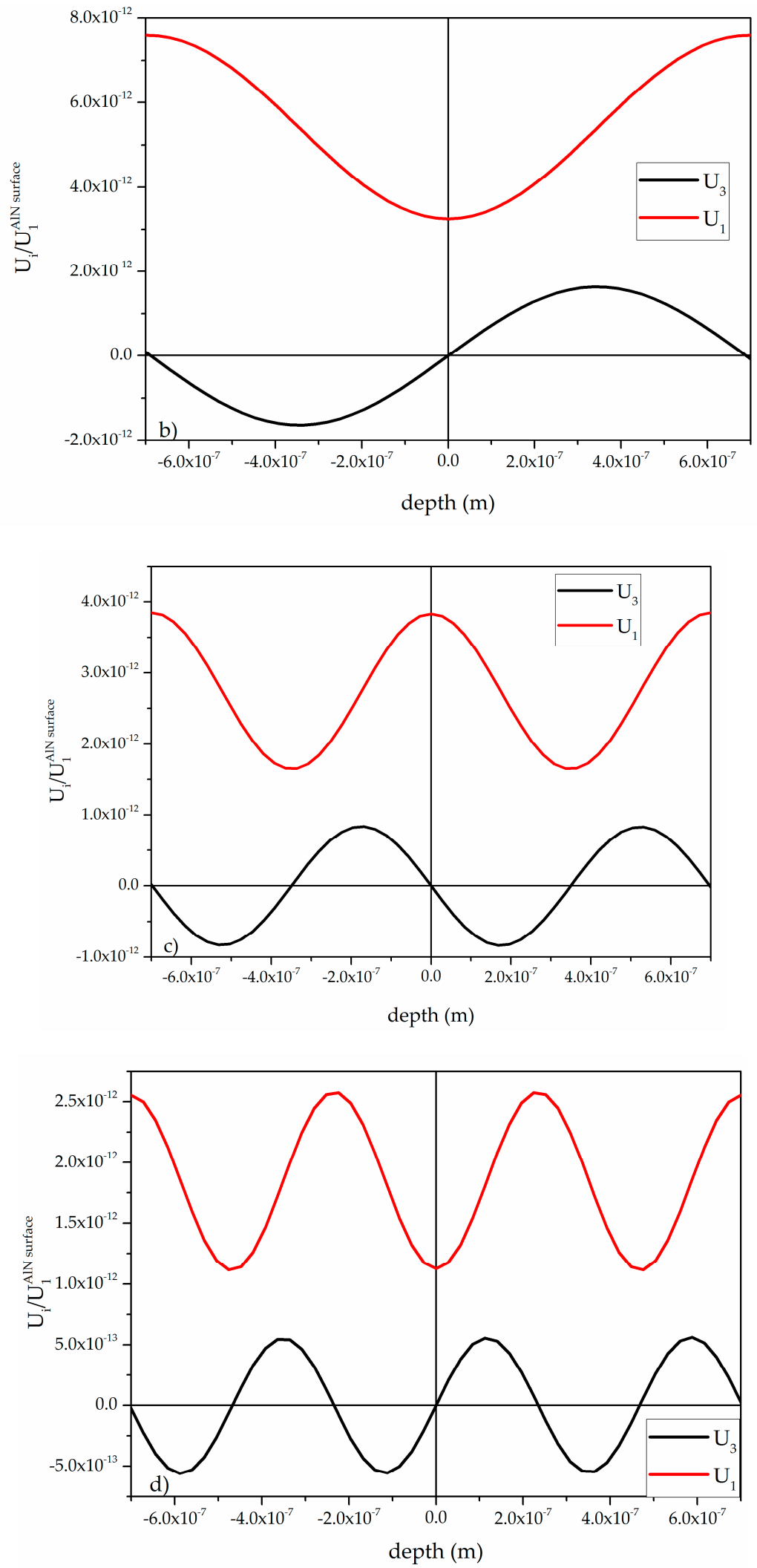

Figure 2. The field profile of the (a) $\mathrm{qS}$; $(\mathbf{b}) \mathrm{qS}_{1}$; (c) $\mathrm{qS}_{2}$; and (d) $\mathrm{qS} 3$ quasi-symmetric Lamb modes.

The four modes frequencies are: 285.54, 5623.85, 11145.2 and $16612.7 \mathrm{MHz}$, respectively. They travel at velocity equal to $10,868.1,11,343.9,11,351.9$, and 11,357.5 m/s, respectively. Generally, one way to obtain a thin AlN suspended membrane consist in the AlN layer deposition onto a silicon wafer covered with a thin $\mathrm{SiN}$ or $\mathrm{SiO}_{2}$ layer; the latter film acts as a stop etching layer during the 
silicon micromachining process. Thus the final suspended membrane is a bilayered membrane (SiN/AlN) that has no symmetry with respect to the mid-plane of the plate: the Lamb modes dispersion curves for this composite plate are expected to be different from those shown in Figure 1 and referred to a single material plate (AlN) [2]. The flat regions are shorter than the previous case and the modes cannot be anymore identified as symmetric or antisymmetric: the modes will be named hereafter with an increasing number. As an example, Figure 3 shows the dispersion curves of the Lamb modes travelling along the composite plate AlN/SiN with total thickness $\mathrm{H}_{\text {total }}=1.6 \mu \mathrm{m}$, being $1.4 \mu \mathrm{m}$ and $0.2 \mu \mathrm{m}$ the AlN and SiN thicknesses.

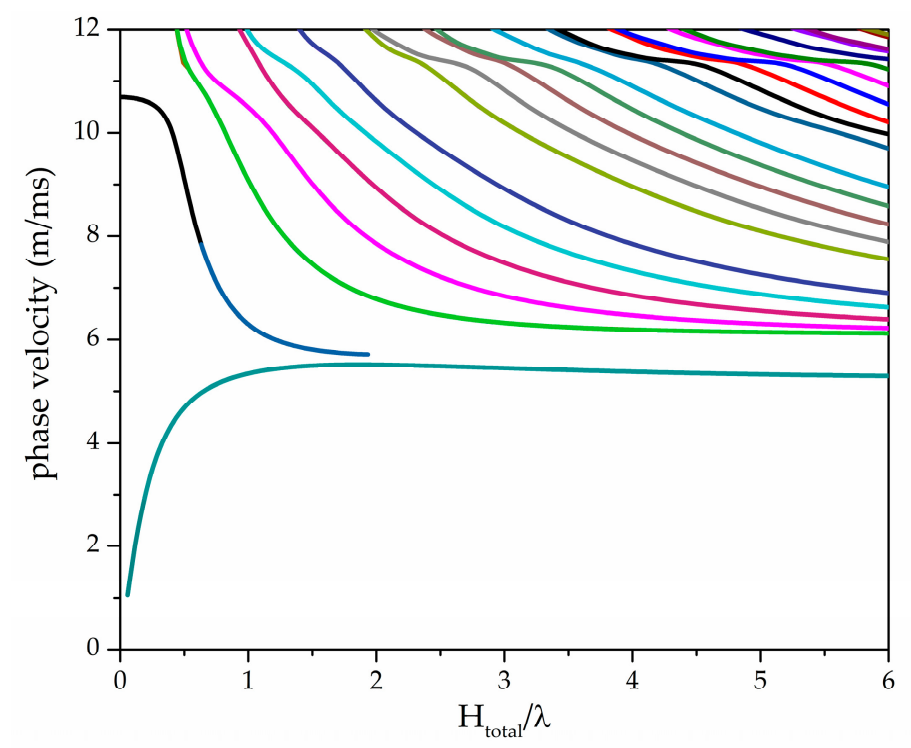

Figure 3. The dispersion curves of the Lamb modes travelling along the composite plate AIN/SiN with total thickness $1.6 \mu \mathrm{m}$, being $1.4 \mu \mathrm{m}$ and $0.2 \mu \mathrm{m}$ the AlN and SiN thicknesses.

Three modes were identified that travel along the SiN/AlN composite plate and that are suitable for liquid sensing applications. Figure $4 \mathrm{a}-\mathrm{c}$ shows the field profile of these three quasi symmetric modes, $\mathrm{qS}_{0}$, $\mathrm{qL} 1$, and $\mathrm{qL} 2$ : the corresponding $\mathrm{H}_{\text {total }} / \lambda$ values are $0.08,0.80$ and 1.6. As it can be seen, $\mathrm{U}_{3}$ is very low with respect to $\mathrm{U}_{1}\left(\mathrm{U}_{3}<<\mathrm{U}_{1}\right)$ on one plate side that is thus the one suitable for contacting a liquid environment.
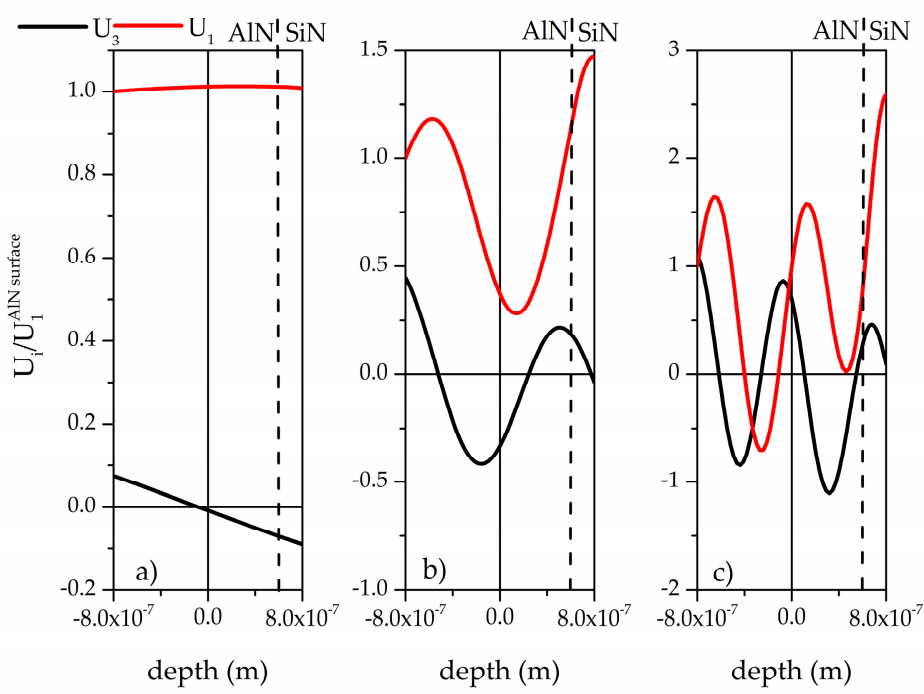

Figure 4. The field profile of the (a) $\mathrm{qS}_{0}$; (b) $\mathrm{qL}_{1}$; and (c) $\mathrm{qL} 2$ modes in air. 
COMSOL FEM Multiphysics software was employed to simulate the three modes propagation along the composite plate with the liquid (water) contacting the plate surface. 2D piezoelectric device simulation with solid mechanics and electrostatic modules was used for eigen-frequency analysis of the composite plate. The number of degrees of freedom to solve for the mode is minimized by providing periodic boundary conditions to the transmitting IDTs which is a one-finger structure with a total width of one $\lambda$. Figure 5 shows the field profile of the three modes in the SiN/AlN plate contacting the liquid environment (water) from the SiN side of the plate. As can be seen, the acoustic energy is confined inside the plate.

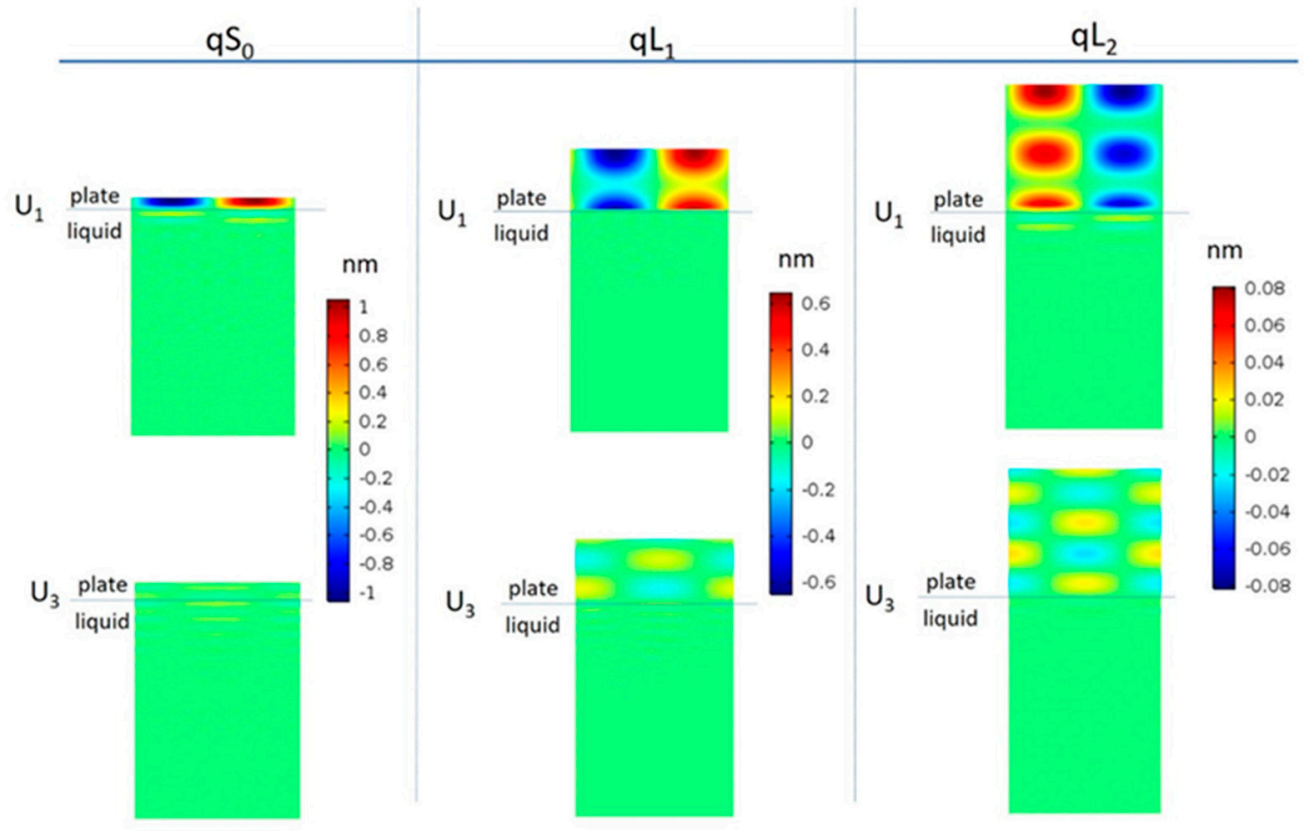

Figure 5. The FEM of the field profile for the $\mathrm{qS}_{0}, \mathrm{qL}_{1}$ and $\mathrm{qL}_{2}$ modes.

\section{The Coupling Efficiency}

The electroacoustic coupling coefficient, $\mathrm{K}^{2}$, physically represents the IDT electrical to acoustic energy conversion efficiency: it is frequency dispersive and strongly affected by the electrical boundary conditions. In the SiN/AlN plate, four piezoelectric coupling configurations can be obtained by placing the IDT at the SiN/AlN interface (SiN-Transducer- Film, STF) or at the AlN surface (SiN- Film-Transducer, SFT), further including a floating metal electrode onto the AlN layer side opposite to that where the IDT is located (SiN-Transducer-Film-Metal and SiN-Metal- FilmTransducer, STFM and SMFT). The four configurations are depicted in Figure 6.
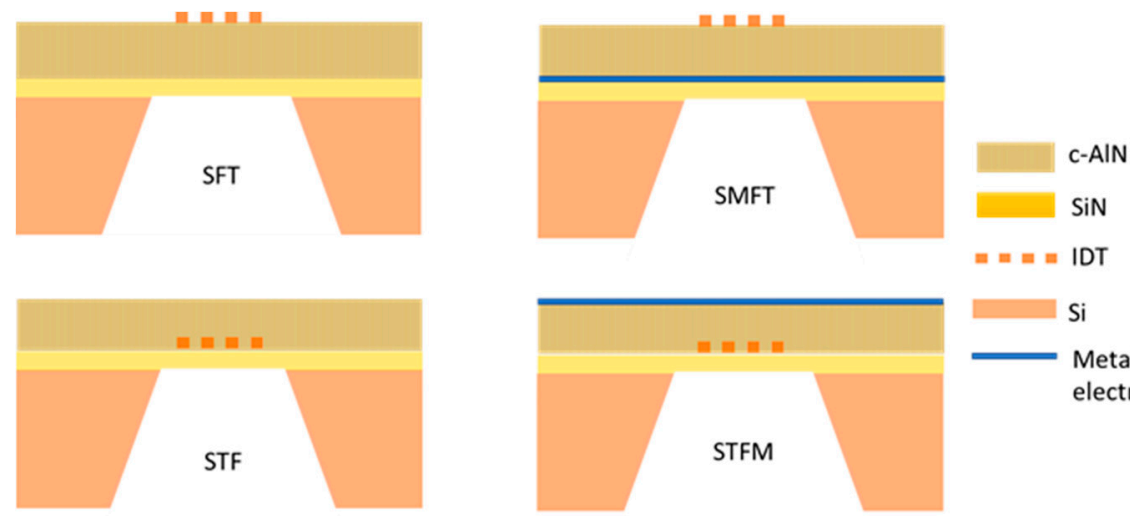

Figure 6. The four coupling configurations: SFT, SMFT, STF and STFM. 
FEM analysis was performed using COMSOL 5.2 to calculate the $\mathrm{K}^{2}$ as $\mathrm{K}^{2} \approx 2\left[\left(\mathrm{vf}_{\mathrm{f}}-\mathrm{vm}_{\mathrm{m}}\right) / \mathrm{vf}\right]$, where $\mathrm{V}_{\mathrm{f}}$ and $\mathrm{V}_{\mathrm{m}}$ are the velocities along the electrically open and shorted surfaces of the AlN film. In Table 1 the $\mathrm{K}^{2}$ of the four coupling configurations are summarized for each mode.

Table 1. The $\mathrm{K}^{2}$ of the four coupling configurations for each mode in the SiN $(200 \mathrm{~nm}) / \mathrm{AlN}(1.4 \mu \mathrm{m})$ plate, for $\lambda=20,2$, and $1 \mu \mathrm{m}$.

\begin{tabular}{ccccc}
\hline \multirow{2}{*}{ Mode/K $(\%)$} & \multicolumn{4}{c}{ Coupling Configuration } \\
\cline { 2 - 5 } & SFT & SMFT & STF & STFM \\
\hline $\mathrm{qS}_{0}$ & $0.22 \%$ & $2.98 \%$ & $0.14 \%$ & $2.90 \%$ \\
$\mathrm{qL} 1$ & $0.91 \%$ & $0.90 \%$ & 0.039 & $0.026 \%$ \\
$\mathrm{qL} 2$ & $0.55 \%$ & $0.55 \%$ & $0.01 \%$ & $0.011 \%$ \\
\hline
\end{tabular}

\section{Lamb Wave Sensor}

\subsection{Mass Sensor}

A mass accumulation on the device surface changes the surface density of the propagating medium, hence resulting in a mode velocity shift. The gravimetric sensitivity $S_{m}$ in air of the three modes on each side of the composite plate was calculated as the relative velocity shift per unit added mass, $\mathrm{S}_{\mathrm{m}}=\left(\mathrm{v}_{\mathrm{b}}-\mathrm{v}_{\mathrm{l}} / \mathrm{v}_{\mathrm{b}}\right) / \mathrm{m}_{\mathrm{am}}$, being $\mathrm{vb}_{\mathrm{b}}$ and $\mathrm{v}_{\mathrm{l}}$ the velocity of the bare and loaded plate, $\mathrm{mam}_{\mathrm{a}}=\mathrm{Q} \cdot \mathrm{d}, \mathrm{Q}$ and $d$ the added mass density and thickness. The $S_{m}$ at the AlN side was equal to $-630,-2400$ and $-2383 \mathrm{~cm}^{2} \mathrm{~g}^{-1}$, while at the SiN side was equal to $-640,-3770$ and $-2425 \mathrm{~cm}^{2} \mathrm{~g}^{-1}$, for the $\mathrm{qS}_{0}, \mathrm{qL} 1$ and $\mathrm{qL}_{2}$ modes, respectively. The gravimetric detection occurring at the AlN or SiN sensing surface of the same plate shows different efficiency as a consequence of a different mode energy confinement at the plate's sides: the highest gravimetric sensitivity is achieved for the plate sensing surface that transmits an higher amount of acoustic energy to the mass loading layer.

\subsection{Viscosity Sensor}

When a liquid contacts the acoustic waveguide, the in-plane particle displacement component of the acoustic mode couples to a very thin viscous boundary layer of thickness $\delta=(2 \eta / \omega \mathrm{Ql})^{0.5}$, where $\eta$ and $\varrho$ are the liquid viscosity and mass density. The viscous liquid was supposed to be a mixture of water and glycerol; the fraction of glycerol by volume ranged from 0 (only water) to 0.53 , and the $\sqrt{\rho \eta_{1}}$ ranged from 0.95 to about $11 \mathrm{~kg} \cdot \mathrm{m}^{-2} \cdot \mathrm{s}^{-0.5}$. The real and imaginary parts of the phase velocity of the three modes were calculated for different concentrations of the water/glycerol mixtures. The relative changes of the phase velocity $\Delta v / v 0$ and the IL as a function of $\sqrt{\rho \eta_{1}}$ are shown in Figure $7 \mathrm{a}, \mathrm{b}$ where it is assumed that the examined glycerol-water mixture contacts the SiN surface of the composite plate.

The qSo mode has a linear IL and relative velocity shift behavior vs. the $\sqrt{\rho \eta_{1}}$ values in the studied viscosity range: its time period ( $2.06 \mathrm{~ns})$ is far larger than the fluid relaxation time $\tau=\eta / \mu$ (where $\mu$ is the liquid shear modulus) for the 0.9 to 11 abscissa value. The $\mathrm{qS}$ o velocity and attenuation sensitivities are $-0.0015 \mathrm{~m}^{2} \mathrm{~s}^{0.5} \mathrm{~kg}^{-1}$ and $0.1038 \mathrm{~dB} / \lambda \mathrm{m}^{2} \mathrm{~s}^{0.5} \mathrm{~kg}^{-1}$. The relative velocity shift of the $\mathrm{qL} 1$ mode is linearly dependent on $\sqrt{\rho \eta_{1}}$ only for low viscosity values, and its slope is reversed for glycerol/water percentage $\geq 20 \%$, as $\tau$ becomes close to the wave period ( $0.2 \mathrm{~ns})$ of the mode. The relative velocity shift of the $\mathrm{qL} 2$ mode is linearly dependent on $\sqrt{\rho \eta_{1}}$ for very low viscosity values, and is reversed for glycerol/water percentage $\geq 10 \%$, as $\tau$ becomes close to the wave period ( $0.099 \mathrm{~ns})$ of the mode. 

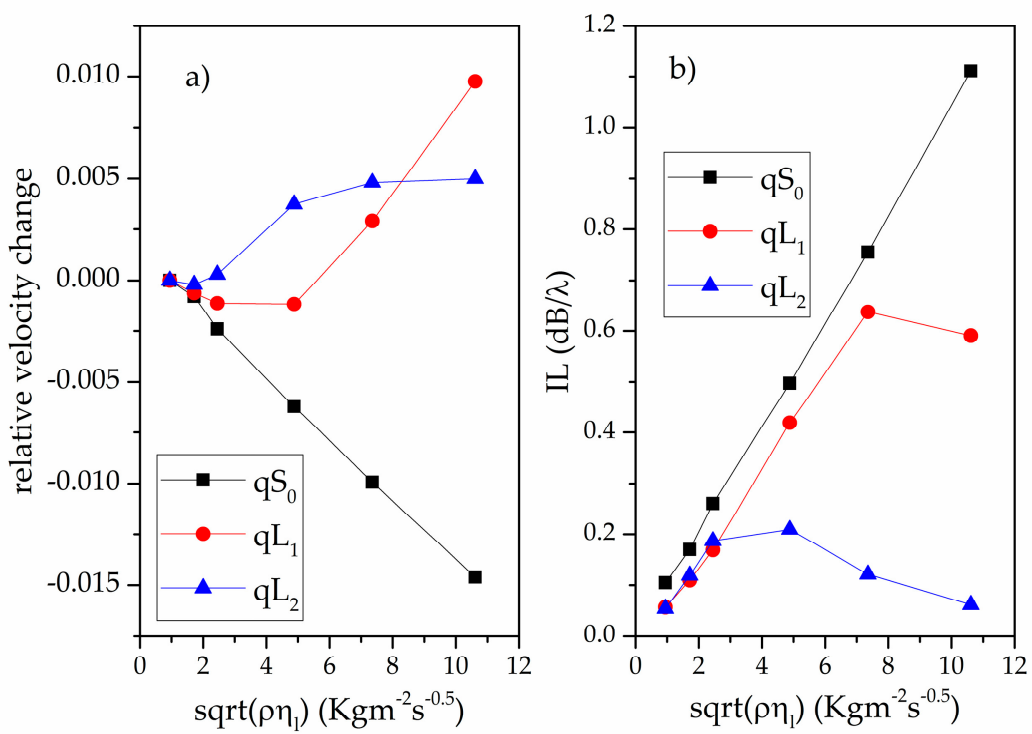

Figure 7. (a) The wave relative velocity change and (b) the IL vs. the square root of the viscous liquid mass density-viscosity product; the water/glycerol mixture is supposed to contact the SiN side of the composite plate.

\section{Works in Progress}

$\mathrm{Cr} / \mathrm{Au}$ IDTs were implemented onto the bare SiN thin suspended membrane $(200 \mathrm{~nm}$ thick, and with the area equal to $1.5 \mathrm{~mm} \times 1.5 \mathrm{~mm}$ ) by electron beam lithography (EBL): the IDTs width of 0.5 $\mu \mathrm{m}$ (equal to $\lambda / 4$ ) was achieved with EBL onto the SiN suspended membrane $(\lambda=2 \mu \mathrm{m})$. A c-AlN layer was deposited by rf magnetron sputtering technique onto a reduced area of the surface of the SiN membrane by using a shadow mask with an opening area of $1 \mathrm{~mm} \times 1 \mathrm{~mm}$, thus an AlN layer was sputtered having an "island-like" structure, as shown in Figure 8.

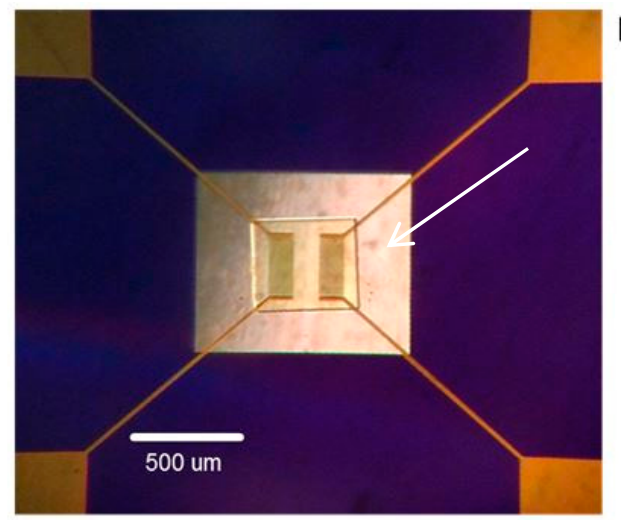

Figure 8. Photo of a couple of IDTs on the SiN/AlN suspended membrane; the blue area is the Si/SiN frame; a white arrow shows the SiN suspended membrane; the central island is the SiN/AlN.

The use of a mask is a method that offers the remarkable advantage to reduce the complexity of the device fabrication process based on several technological steps, including the silicon substrate surface or bulk micromachining and the etching of a sacrificial layer to release the membrane.

\section{Conclusions}

The propagation of quasi Lamb modes along SiN/AlN composite plates have been theoretically investigated. The displacement profiles, phase velocities, and the electromechanical coupling coefficients of four coupling configurations have been calculated, specifically addressing the design 
of enhanced-coupling, microwave frequency sensors for liquid environments. The IL and velocity changes of the $\mathrm{qS}_{0}, \mathrm{qL}_{1}$, and $\mathrm{qL}_{2}$ modes when contacting a viscous Newtonian liquid have been calculated for different viscosities. The SiN/AlN-based sensors are proven to achieve remarkable performances (high sensitivity and enhanced coupling efficiency) that are important prerequisites for the design of future devices to be used in the context of chemical, biological, and physical quantities detection.

Acknowledgments: This study has received funding from the European Union's Horizon 2020 Research and Innovation Programme under the Marie Sklodowska-Curie Grant Agreement No. 642688.

Author Contributions: C. Caliendo wrote the paper and made the Disperse simulations; E. Giovine optimized and performed the EBL process onto the SiN membrane; M. Hamidullah performed the FEM calculations; C. Caliendo and M. Hamidullah sputtered the AlN layer.

Conflicts of Interest: The authors declare no conflict of interest.

\section{References}

1. Caliendo, C. Longitudinal Modes along Thin Piezoelectric Waveguides for Liquid Sensing Applications. Sensors 2015, 15, 12841-12856, doi:10.3390/s150612841.

2. Caliendo, C.; Hamidullah, M.; Laidoudi, F. Amorphous SiC/c-ZnO-Based Quasi-Lamb Mode Sensor for Liquid Environments. Sensors 2017, 17, 1209, doi:10.3390/s17061209.

(c) 2018 by the authors. Licensee MDPI, Basel, Switzerland. This article is an open access article distributed under the terms and conditions of the Creative Commons Attribution (CC BY) license (http://creativecommons.org/licenses/by/4.0/). 